\title{
Partially Rigid 3D Registration of Flexible Tissues in High Resolution Anatomical MRI
}

\author{
Stepan Pazekha ${ }^{1}$, Darius Gerlach ${ }^{2}$, Uwe Mittag ${ }^{2}$, Rainer Herpers ${ }^{1,3,4}$ \\ ${ }^{1}$ Institute for Visual Computing, HBRS Sankt Augustin, Germany \\ ${ }^{2}$ Institut für Luft- und Raumfahrtmedizin, DLR Köln, Germany \\ ${ }^{3}$ Dep. of Comp. Sci. and Eng., York University, Toronto, Canada \\ ${ }^{4}$ Fac. of Computer Science, University of New Brunswick, Canada \\ stepan.pazekha@smail.inf.h-brs.de
}

\begin{abstract}
This contribution introduces a method for partially rigid 3D registration of high resolution magnetic resonance (MR) images of the eye globes (EG) and the optic nerve sheaths (ONS) based on the reconstruction of their $3 \mathrm{D}$ models. Conventional registration methods do not preserve anatomical structures in such a way that quantitative anatomical comparisons could be computed. Therefore, iterative closest points (ICP) registration method has been extended to enable partial rigid registration (PICP) of flexible tissue structures within certain spatially limited areas. The results of the proposed approach are compared with the non-linear registration method ART. It was shown that PICP approach considerably improved the matching quality of local tissue and preserved anatomical structure at the same time.
\end{abstract}

\section{Introduction}

The optic nerve sheath is the protective layer around the optic nerve and is filled with the cerebrospinal fluid. Mader et al. [1] studied seven astronauts before and after a 6 month space mission. Among several ophthalmic findings, the authors report on two types of structural changes: an increase in the optic nerve sheath diameter and a flattening of the back wall of the eye globe $[2,3]$. A series of laboratory experiments with 10 subjects were conducted to check the hypothesis. Each experiment consisted of two measurement sessions. During the first session the subjects were placed in a horizontal (supine) position. To simulate the micro-gravity conditions, the subjects were afterwards put in a head down tilted (HDT) position (random slope of $6^{\circ}, 12^{\circ}$ or $18^{\circ}$ ). After 5 hours in the HDT position, the second measurement session was performed. Each session included acquiring a set of high resolution (image pixel size $\sim 0.4 \times 0.4 \mathrm{~mm}$, distance between image slices $\sim 2 \mathrm{~mm}$ ) $\mathrm{T}^{*}$ weighted magnetic resonance (MR) images of the eye regions in 3 directions: sagittal, coronal and transverse (Fig. 1). The structural changes in the eye globes (EG) and the optic nerve sheaths (ONS) had to be investigated afterwards by comparing data from supine and HDT measurements. Image registration has been applied to find a one-to-one transform (mapping) between source, $\mathrm{I}_{\mathrm{s}}$, and target, $\mathrm{I}_{\mathrm{t}}$, volumetric images. However, the 
particular algorithm for such transform reconstructions is highly dependent on the goal of registration [4, 5]. Two approaches, Automatic Registration Toolbox (ART) and Symmetric image Normalization (SyN) [6], have demonstrated similarly high performance among the other evaluated algorithms, thus ART has been considered and evaluated in our work. However, these high quality non-linear registration methods do not preserve any anatomical structures of the tissues depicted in our images. Moreover, these methods are able to perform registration of unidirectional MR images only. This work proposes a partially rigid registration approach based on the reconstruction of the $3 \mathrm{D}$ model of the flexible tissue (the EG and the ONS). This approach utilizes three cutting directions of MR images for model construction and builds a transformation for the groups of voxels of the anatomy structure that must be preserved. A new evaluation metric is developed to compare the results of proposed method with a non-linear approach (ART).

\section{Segmentation and registration}

The anatomical structures of interest in this work were the back wall of the EG and the $5 \mathrm{~mm}$ long part of the ONS attached to the EG because the evidence of diameter increase was found in this part. To compare these structures in different images we extend the semi-automatic segmentation approach of Singh et al. [7] to make it fully automatic for our data.

\subsection{Segmentation of the eye globe and the optic nerve sheath}

The sagittal images were acquired in such a way that only one EG region was captured. As can be seen in the example images (Fig. 1), the EG region is represented by a circular shape of high intensity. The images of the sagittal plane are therefore preprocessed using the circle Hough transform (CHT). The $\mathrm{k}$-means clusterization is used to separate the positive detections from the false ones. The cluster with the biggest number of centroids is chosen as the candidate for the EG detection. The shape of the EG is not exactly spherical that is why we use the flood-fill algorithm to adapt the final segment. A binary segmentation mask is initialized around each of the circle centers. The flood-fill algorithm then is implemented as follows. Let $\mathrm{M}=\left\{\mathbf{x}_{\mathbf{i}}, \mathrm{i} \in \mathbf{N}\right\}$ represent a set of points included

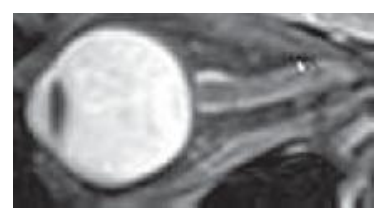

(a) Sagittal

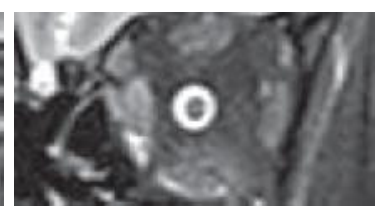

(b) Coronal

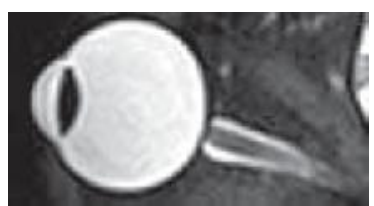

(c) Transverse

Fig. 1. Sample midcuts of the MR images of the eye regions in three directions: sagittal (a), coronal (b) and transverse (c). The structures of interest are the eye globes and the optic nerve sheaths because structural changes were reported there. 
in the initial binary mask and $B$ represent a set of boundary points that can be given as $\mathrm{B}=\{\mathbf{y}: \mathbf{y} \notin \mathrm{M} \wedge \exists \mathbf{z}: 0.5<\|\mathbf{z}-\mathbf{y}\|<1.5 \wedge \mathbf{z} \in \mathrm{M}\}$. Let also I(x) denote an image intensity value at pixel $\mathbf{x}$. A boundary point $\mathbf{y} \in \mathrm{B}$ is added to the mask $M$ if the following rule applies

$$
-\frac{\mathrm{I}(\mathbf{y})}{\mathrm{I}(\mathbf{y})+\mu} \log _{2}\left(\frac{\mathrm{I}(\mathbf{y})}{\mathrm{I}(\mathbf{y})+\mu}\right)-\frac{\mu}{\mathrm{I}(\mathbf{y})+\mu} \log _{2}\left(\frac{\mu}{\mathrm{I}(\mathbf{y})+\mu}\right)>\mathrm{t}
$$

where $\mu$ is the mean intensity of the points in $\mathrm{M}$ and $\mathrm{t}$ is the intensity similarity threshold. Each boundary point is checked according to the described rule until none of the points $\mathbf{y} \in \mathrm{B}$ can be added to $\mathrm{M}$. After the flood-fill algorithm is applied to each image slice, we obtain a $3 \mathrm{D}$ binary matrix of the segmentation mask.

This information, computed in the sagittal images so far, is used to support the segmentation of the EG in the transverse and the coronal image sequences as well. The support is realized by projecting the sagittal binary mask voxels to transverse and coronal image planes. The projected voxels are used as the initial mask voxels for repeated flood-fill algorithm application.

The ONS segmentation is realized with the same algorithm as described above, however, the processing starts at the coronal images. The CHT algorithm is applied to detect the images containing ONS (Fig. 1(b)). After filtering out false detections the voxels from the top $5 \%$ intensity quantile form the initial binary mask for flood-fill algorithm application. The resulting binary masks of the ONS in coronal planes support the segmentation of the ONS in the sagittal and the transverse image sequences.

\subsection{Partially rigid $3 \mathrm{D}$ registration}

In the next processing step the gradient across all three image directions is calculated. As a result, only mask edges remain in the binary images. In our case MR images were acquired in DICOM format which provides transformation quaternions from image coordinates to reference coordinate system (RCS) for each image plane. We use these transformations to recover the coordinates of each pixel belonging to the edges of the binary masks. The resulting point cloud represents the 3D model of the EG and the ONS separately. Due to the sparsity of the slice-to-slice distance $(\sim 2 \mathrm{~mm})$ with respect to the image resolution of $\sim 0.4 \times 0.4 \mathrm{~mm}$ and the small size the anatomical structures of interest (ONS) this approach allowed to generate a consistent 3D model of the EG and ONS which combines the anatomical information from all three imaging directions simultaneously.

Rigid registration methods allows to preserve structural properties of the objects, but doesn't allow flexible objects to be robustly registered. However, by selecting correspondent parts of objects to be registered that can be assumed to be rigid, it is possible to apply the registration independently to each pair of correspondent parts. We use the iterative closest point (ICP) algorithm by Besl and McKay [8] to implement partial rigid registration of the 3D models 
(PICP) of the EG and the ONS. The EG area and the $5 \mathrm{~mm}$ long piece of the ONS following the back wall of the eye are assumed to be rigid objects. Utilizing the separated $3 \mathrm{D}$ models from the segmentation stage the rigid registration is independently applied to the pairs of the EG and ONS models.

\section{Evaluation strategy}

To evaluate the proposed 3D registration and compare its performance with the non-linear ART registration, a metric based on volume overlap estimation was developed. Due to irregularity of our MR data resolution $(\sim 0.4 \times 0.4 \times 2 \mathrm{~mm})$ and nature of our 3D model, composed of multi-directional image data, conventional volume estimation metrics based on voxel counting are ineffective. Let $\mathrm{m}_{\mathrm{i}}=$ $\left(\mathrm{x}_{\mathrm{i}}, \mathrm{y}_{\mathrm{i}}, \mathrm{z}_{\mathrm{i}}\right)^{\mathrm{T}}$ represent a $3 \mathrm{D}$ point of the eye model, then $\mathrm{M}=\left(\mathrm{m}_{1}, \mathrm{~m}_{2}, \ldots, \mathrm{m}_{\mathrm{N}}\right)$ represent all points of the model. Also let $\mathrm{R}(\theta)$ be a rotation matrix, then the projection of the model to the plane of rotation can be introduced as

$$
P(\theta)=\left(\begin{array}{lll}
1 & 0 & 0 \\
0 & 1 & 0
\end{array}\right) \mathrm{R}(\theta) \mathrm{M} ; \mathrm{R}(\theta)=\left(\begin{array}{ccc}
\cos (\theta) & 0 & \sin (\theta) \\
0 & 1 & 0 \\
\sin (\theta) & 0 & \cos (\theta)
\end{array}\right)
$$

with $\mathrm{p}_{\mathrm{i}}=\left(\mathrm{x}_{\mathrm{i}}, \mathrm{y}_{\mathrm{i}}\right)^{\mathrm{T}}$ representing $2 \mathrm{D}$ coordinates of the projected points. Also let $S(\cdot)$ represent a cumulative area of the Delaunay triangulation, then the volume of the $3 \mathrm{D}$ point cloud could be estimated as $\mathrm{V}_{\mathrm{M}}=\sum_{\theta=0}^{\pi} \mathrm{S}(\mathrm{P}(\theta))$. In each registration case, the two models have to be matched. The ratio between the volume estimation of both models and the volume estimation of one of the models gives a volume overlap score $\mathrm{V}_{\mathrm{sc}}=\frac{\mathrm{V}_{\mathrm{M}_{1}}}{\mathrm{~V}_{\mathrm{M}_{1}, \mathrm{M}_{2}}}$. In addition to the volume overlap score, we calculate the angles between image planes before and after registration. To use the volume overlap score metric for the results of non-linear ART registration, we warp the resultant displacement field with the 3D binary mask of the transverse plane. The 3D model of the EG and the ONS is then reconstructed using only the transformed binary mask of the transverse plane due to the inability of ART to process multi-directional MR images simultaneously.

\section{Results}

A database of MR images acquired from 10 subjects was processed using the proposed segmentation approach. A sample segmentation result is shown in Fig. 4(a). After we obtain the segmentation, the 3D model of the EG is reconstructed using the binary masks from all three imaging directions. A sample reconstruction for two correspondent sets of MR images of the same eye are shown in Fig. 4(b).

The PICP approach splits the 3D model into the EG part and the ONS part and registers them separately. Thus, the results with the ART approach for each of the parts are compared. The volume overlap scores calculated for 
Table 1. Statistics on the volume overlap score for the ART and PICP methods in the eye globes (EG) and the optic nerve sheaths (ONS) areas. The mean and standard deviation (SD) values were calculated over all 10 subjects separately for left and right eyes.

\begin{tabular}{lllllllll}
\hline \multicolumn{3}{c}{ Left eye } & & \multicolumn{5}{c}{ Right eye } \\
& EG & & ONS & & EG & & ONS & \\
& Mean & SD & Mean & SD & Mean & SD & Mean & SD \\
\hline ART & 0.9117 & 0.0912 & 0.7826 & 0.1297 & 0.9552 & 0.0092 & 0.8348 & 0.0764 \\
PICP & 0.9675 & 0.0081 & 0.8560 & 0.0599 & 0.9737 & 0.01 & 0.8615 & 0.07022 \\
\hline
\end{tabular}

the EG show similarly high results for both approaches. However, in the ONS region the proposed PICP approach performs slightly better than the non-linear approach (Tab. 1). The mean scores in the ONS part are not high enough to state that there were any structural changes in it. The spatial orientation of the image planes after registration can be calculated as the relative angle between them. The results for the relative transverse image plane calculation are given in Tab. 2. The table shows that the relative angles between transverse planes before registration are nonzero. The relative angles appear as a result of a process of MR images acquisition where the operator has to adjust the orientation of the cutting planes manually prior to each acquisition. However, after the application of the ART segmentation, all relative angles between transverse planes become zero. For the PICP approach the relative angles remain nonzero even after the registration application.

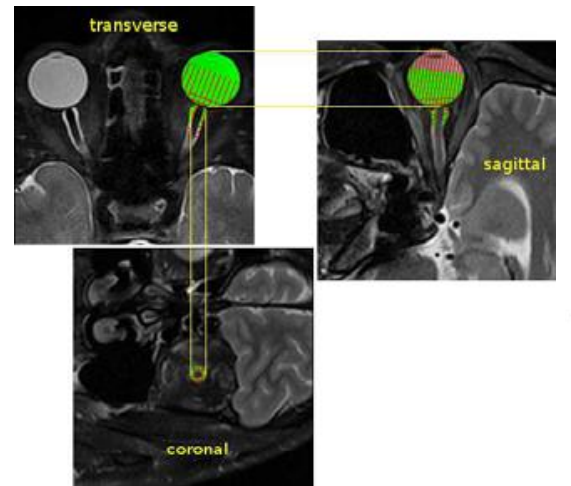

(a) Segmentation

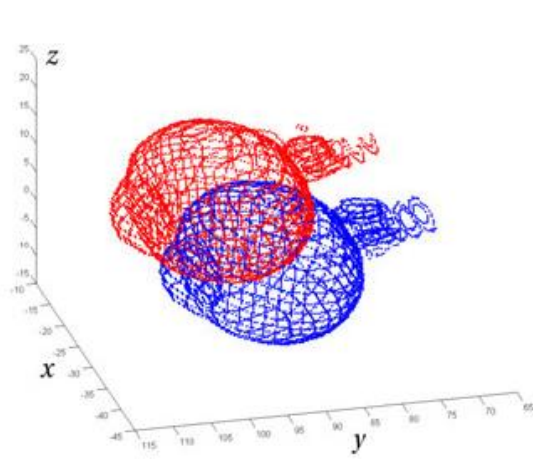

(b) $3 \mathrm{D}$ reconstruction

Fig. 2. Sample segmentation of the eye globe and the optic nerve in the midcuts of MR images (a). The binary mask is marked in green, points of the binary mask projected to the correspondent plane intersection lines are marked in red. Correspondent planes are marked with yellow. The original orientation of the two reconstructed 3D models to be registered (b) of the same eye from suppine (red) and HDT (blue) measurements in DICOM reference coordinate system. The blue model is shifted and rotated with respect to the red model due to HDT intervention. 
Table 2. Angle in degrees between transverse planes before and after registration for PICP method: eye globe (EG), optic nerve sheath (ONS). ART approach forces the image planes to become parallel (zero angle), meaning that the actual anatomical structure is distorted in order to be registered. PICP approach preserves the anatomical structure by applying only a rigid type of registration.

\begin{tabular}{lrrrrrrrrrr}
\hline Experiment No. & 1 & 2 & 3 & 4 & 5 & 6 & 7 & 8 & 9 & 10 \\
\hline Initial & 10.837 & 2.268 & 1.938 & 14.385 & 21.307 & 10.280 & 3.846 & 6.155 & 7.084 & 2.782 \\
PICP EG & 7.399 & 1.290 & 3.540 & 14.969 & 21.272 & 7.852 & 3.862 & 5.180 & 4.755 & 0.829 \\
PICP ONS & 11.566 & 2.393 & 23.638 & 7.484 & 3.233 & 12.886 & 9.411 & 5.902 & 11.664 & 6.730 \\
\hline
\end{tabular}

\section{Conclusions}

This work proposes a partially rigid $3 \mathrm{D}$ registration approach based on the ICP algorithm (PICP). We introduce a metric to quantitatively evaluate the performance of the proposed registration method and compare it with the non-linear registration approach (ART). PICP is found to perform better than ART for cases like ours when anatomical structures need to be preserved to compute quantitative measurement out of MR data with high image resolution $(\sim 0.4 \times 0.4 \mathrm{~mm})$ but low slice-to-slice resolution $(\sim 2 \mathrm{~mm})$. PICP is also able to preserve the geometrical structure of the registered objects by incorporating the data from several image planes. It was shown that PICP keeps the quality of the registration high by splitting flexible objects into parts and applying rigid transformations independently to each of them.

\section{References}

1. Mader TH, Gibson CR, Pass AF, et al. Optic disc edema, globe flattening, choroidal folds, and hyperopic shifts observed in astronauts after long-duration space flight. Ophthalmol. 2011;118(10):2058-69.

2. Kramer LA, Sargsyan AE, Hasan KM, et al. Orbital and intracranial effects of microgravity: dindings at 3-T MR imaging. Radiology. 2012;263(3):819-27.

3. Marshall-Bowman K, Barratt MR, Gibson CR. Ophthalmic changes and increased intracranial pressure associated with long duration spaceflight: an emerging understanding. Acta Astronaut. 2013;87(0):77-87.

4. Gholipour A, Kehtarnavaz N, Briggs R, et al. Brain functional localization: a survey of image registration techniques. IEEE Trans Med Imaging. 2007;26(4):427-51.

5. Klein A, Andersson J, Ardekani BA, et al. Evaluation of 14 nonlinear deformation algorithms applied to human brain $\{\mathrm{MRI}\}$ registration. Neuroimage. 2009;46(3):786802.

6. Avants BB, Epstein CL, Grossman M, et al. Symmetric diffeomorphic image registration with cross-correlation: evaluating automated labeling of elderly and neurodegenerative brain. Med Image Anal. 2008;12(1):26-41.

7. Singh KD, Logan NS, Gilmartin B. Three-dimensional modeling of the human eye based on magnetic resonance imaging. Invest Ophthalmol Vis Sci. 2006;47(6):2272.

8. Besl PJ, McKay ND. Method for registration of 3-D shapes. Proc SPIE. 1992;1611:586-606. 